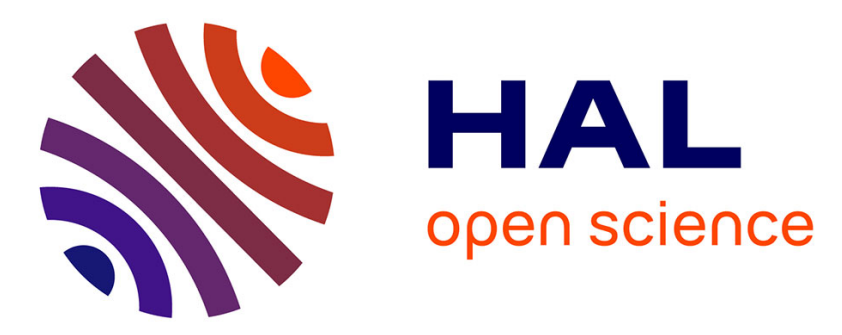

\title{
How to solve inverse scattering problems without knowing the source term: a three-step strategy
}

Marie Graff, Marcus J. Grote, Frédéric Nataf, Franck Assous

\section{To cite this version:}

Marie Graff, Marcus J. Grote, Frédéric Nataf, Franck Assous. How to solve inverse scattering problems without knowing the source term: a three-step strategy. Inverse Problems, 2019, 35 (10), pp.104001. 10.1088/1361-6420/ab2d5f . hal-02002931

\section{HAL Id: hal-02002931 https://hal.science/hal-02002931}

Submitted on 31 Jan 2019

HAL is a multi-disciplinary open access archive for the deposit and dissemination of scientific research documents, whether they are published or not. The documents may come from teaching and research institutions in France or abroad, or from public or private research centers.
L'archive ouverte pluridisciplinaire HAL, est destinée au dépôt et à la diffusion de documents scientifiques de niveau recherche, publiés ou non, émanant des établissements d'enseignement et de recherche français ou étrangers, des laboratoires publics ou privés. 


\title{
How to solve inverse scattering problems without knowing the source term: a three-step strategy
}

\author{
Marie Graff ${ }^{1 *}$, Marcus J. Grote ${ }^{2}$, Frédéric Nataf ${ }^{3,4,5}$, Franck Assous ${ }^{6}$ \\ ${ }^{1}$ Department of Mathematics, University of Auckland, Private Bag 92019, Auckland 1142, New Zealand. \\ marie.graff@auckland.ac.nz \\ 2 Departement für Mathematik und Informatik, Universität Basel, Spiegelgasse 1, CH-4051 Basel, \\ Switzerland. marcus.grote@unibas.ch \\ ${ }^{3}$ CNRS, UMR 7598, Laboratoire Jacques-Louis Lions, F-75005, Paris, France. nataf@ljll.math.upmc.fr \\ ${ }^{4}$ UPMC Univ. Paris 06, UMR 7598, Laboratoire Jacques-Louis Lions, F-75005, Paris, France. \\ ${ }^{5}$ INRIA Rocquencourt, Alpines, BP 105, 78153 Le Chesnay Cedex, France. \\ ${ }^{6}$ Department of Mathematics, Ariel University, 40700, Ariel, Israel. franckassous55@gmail.com
}

January 2019

\begin{abstract}
The solution of inverse scattering problems always presupposed knowledge of the incident wavefield and require repeated computations of the forward problem, for which knowing the source term is crucial. Here we present a three-step strategy to solve inverse scattering problems when the time signature of the source is unknown. The proposed strategy combines three recent techniques: (i) wave splitting to retrieve the incident and the scattered wavefields, (ii) time-reversed absorbing conditions $(T R A C)$ for redatuming the data inside the computational domain, (iii) adaptive eigenspace inversion (AEI) to solve the inverse problem. Numerical results illustrate step-by-step the feasibility of the proposed strategy.
\end{abstract}

\section{Introduction}

Parameter estimation plays a crucial role in imaging whether in the medical field or in seismic exploration, and remains an active subject of research, with many applications such as tumor detection or stroke prevention in the case of medical imaging [39, 37]. In geophysics, seismic imaging is used as a tool for exploring subsoil for oil, gas or other deposits [48, 15]. From the mathematical point of view, parameter estimation can be written as a PDE-constrained optimization problem [45, 26, 29], which tries to minimize the misfit between the recorded data and the reconstruction obtained by an estimated parameter. At each step of the optimization process, this estimation is updated to get closer to the original parameter to recover.

However, the PDE-constrained optimization problem needs to repeatedly solve the forward problem, which requires the source that illuminated the medium. Even though it is often assumed that the source is known, some applications do not provide that information. Sometimes, an approximate location of the source may be known, but the time history or signature of the resulting incident signal is hardly available. For instance, in [41] the authors consider transcranial ultrasonic imaging with cavitation bubbles induced by ultrasound pulses. The collapse of the bubble generates a small shock wave recorded by a transducer array. In this example, the bubble's signature is clearly unknown. Similarly, in photoacoustic imaging, the quantity of interest is the time history of the pressure wave generated by an unknown source resulting of transient thermoelastic expansion from laser pulses [44].

\footnotetext{
${ }^{*}$ Earlier known as Marie Kray.
} 
In this paper, we aim at solving an inverse scattering problem when the time signature of the source is unknown. Let us assume that we wish to recover a scatterer, $\mathcal{O}$ in Fig. 1, from boundary measurements recorded on $\Gamma$, which encloses the computational domain $\Omega$. We know that inclusion $\mathcal{O}$ was initially illuminated by a source located at $x_{s r c}$. However, the time history of the source is not known. Here we propose a decoupled approach where we first virtually move the recorded total field into a zone (redatuming) close to the scatterer and not containing the source. Then we solve the inverse problem to recover the obstacle only. In principle, the purpose of the inverse problem could be extended to recovering the source characteristics together with the parameters of the scatterer. Nevertheless, this happens to increase the complications by multiplying the unknowns to recover: a time signal (the source signature) and a velocity field (the scatterer). Our approach enables us to circumvent this difficulty by separating the reconstruction of the scatterer from the knowledge of the source.

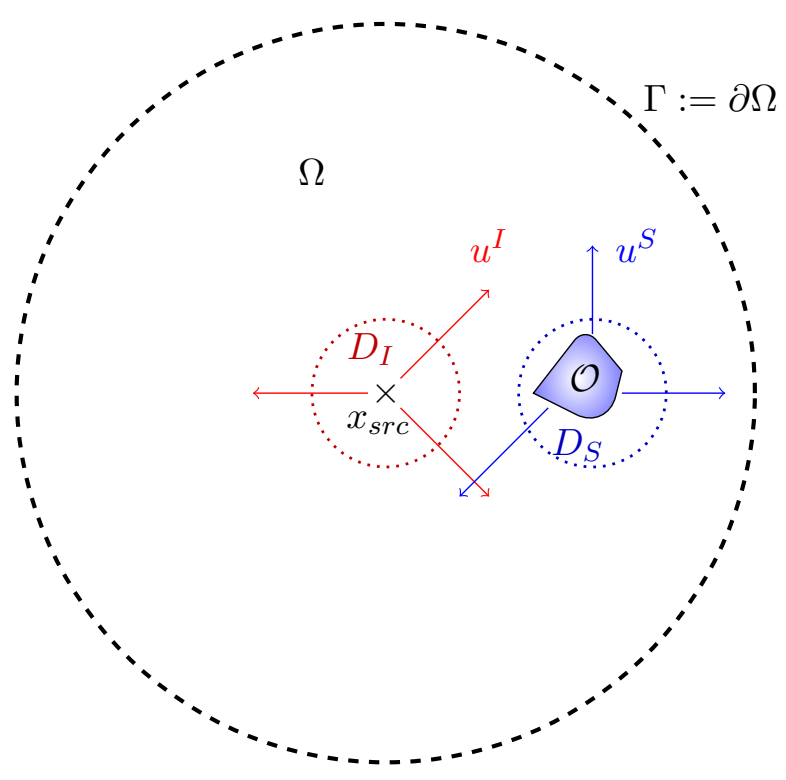

Figure 1: Geometry of the domain $\Omega$ containing a source located at $x_{\text {src }}$ illuminating an obstacle $\mathcal{O}$ to image from measurements of $u=u^{I}+u^{S}$ on the external boundary $\Gamma$

We propose to perform a redatuming of the total field from the boundary $\Gamma$ to a zone close to the scatterer $\mathcal{O}$ and that surrounds it. To do so, our approach is based on the splitting of the total field into a sum of two fields $u=u^{I}+u^{S}$ where $u^{I}$ (resp. $u^{S}$ ) satisfies the homogeneous wave equation except in a zone $D_{I}$ around the source (resp. except in a zone $D_{S}$ around the obstacle $\mathcal{O}$ ), see Figure 1 . First, we shall split the signal on the boundary $\Gamma$ by the method developed in $[23,24,5]$. Then using twice the TRAC method $[3,4,2]$, we shall compute the field $u^{I}$ in $\Omega \backslash D_{I}$ and the $u^{S}$ in $\Omega \backslash D_{S}$. By summing these two fields where it makes sense, we shall recover the total field $u$ everywhere in $\Omega \backslash\left(D_{I} \cup D_{S}\right)$.

The goal of wave splitting is to isolate different components of a complex wavefield, which we know is the result of the sum of several signals $[47,20,1,5]$. In the context of our work, the total wavefield recorded by the transducers is actually the sum of the incident wavefield, which coincides with $u^{I}$, and the scattered wavefield corresponding to $u^{S}$. To split $u^{I}$ from $u^{S}$, various works have been developed, for instance, in the frequency domain [7, 19], or the time domain [43] using Fourier transform. Our method [23, 24] though is local and works directly in the time-dependent domain without Fourier transform. Once wave splitting has been performed, we finally get $u^{I}$ and $u^{S}$ separately on $\Gamma$.

The TRAC method has been derived from Time Reversal techniques [18, 17]. The main idea was to propose a way to reconstruct numerically a wavefield from time reversed measurement data where we actually include a numerical sink. Thence, we set the time reversed absorbing conditions on the artificial boundary of the sink. For instance to reconstruct the incident wave, we remove a small ball $D_{I}$ 
around the location of the source and perform the TRAC algorithm outside this ball, in $\Omega \backslash D_{I}$, from $u^{I}$ on $\Gamma$ recovered by wave splitting. Naturally, no obstacle is involved in this reconstruction. The medium is assumed constant and homogeneous. Similarly, we also back-propagate the scattered wavefield using the TRAC method in the constant and homogeneous domain $\Omega \backslash D_{S}$. Thereby we know the total field, sum of redatumed $u^{I}$ and $u^{S}$, in the vicinity of $D_{S}$ and thus solve the inverse problem for the unknown parameter inside $D_{S}$.

To solve the inverse problem from the redatumed data, many methods are available $[30,42,32$, 27]. In this paper, we choose to solve the inverse problem by performing the Adaptive Eigenspace Inversion (AEI) method [14, 13, 12, 22, 25]. Inverse problems are often modeled by PDE-constrained optimization [26], usually nonlinear, convex and ill-posed $[8,11]$. To overcome the ill-posedness of the optimization problem, regularization is usually added [45, 31], like for instance through a Tikhonov [46, 33, 34] or Total Variation (TV) [16] penalty term, the latter being also widely used in image processing to help increase resolution $[9,10,40]$. In AEI, we actually use regularization by discretization [31, 14] and span the unknown parameter in the eigenfunction basis of a well-chosen elliptic operator.

To the best of our knowledge, this is the first time that these three recent methods are judiciously combined to solve a time-dependent inverse scattering problem, when the source signature is unknown. In fact, these three steps are necessary to enable the solution of the inverse problem. The rest of our paper is structured as follows: Section 2 recollects the theory of the three methods. In section 2.1, we recall the main equations and the principle of wave splitting [24]. In section 2.2, we write the TRAC method [4, 2] to perform redatuming on the total field to reduce the computational domain. Finally, Section 2.3 presents the AEI method to solve the inverse problem for the time-dependent equation [13, 22]. Then Section 3 displays two numerical examples to illustrate each step of our strategy. In section 3.1, we start with a smooth simple elliptic obstacle. Then, we propose a more complex example with a fish-shaped obstacle.

\section{A three-step strategy}

We wish to recover an unknown obstacle buried in a homogeneous medium from total field measurements yet without the knowledge of the source term. Only the location of the source, but not its time history, is assumed to be known. The total wavefield $u$, the sum of the incident wavefield $u^{I}$ and the scattered wavefield $u^{S}$, is recorded during a time interval $[0, T]$ on a circular array $\Gamma:=\partial \Omega$ surrounding the area of interest $\Omega$. It satisfies the wave equation:

$$
\left\{\begin{aligned}
\frac{\partial^{2} u}{\partial t^{2}}-\nabla\left(c^{2}(x) \nabla u\right) & =f(t) \delta\left(x-x_{s r c}\right), & & \text { in }(0, T) \times \Omega, \\
u & =0, & & \text { in } \Omega \text { at } t=0, \\
\frac{\partial u}{\partial t} & =0, & & \text { in } \Omega \text { at } t=0 .
\end{aligned}\right.
$$

Hence, we shall assume that the time signature $f(t)$ is not known, whereas the location $x_{s r c}$ is approximatively known. Moreover, we assume that the source starts emitting only after recording is initiated and that the remaining signal inside $\Omega$ is negligible after $t=T$. The buried obstacle corresponds to variations in propagation speed $c$, which is assumed homogeneous and constant outside the obstacle.

Solution strategies for inverse problems usually require precise knowledge of the source to compute the forward wavefield. In this work however, we shall not make use of that information. Therefore, we wish to virtually move the measured total field to a zone close to the obstacle and not containing the source. To do so, we apply first wave splitting [23, 24] to extract the incident and the scattered components from the recorded total wavefield, and then we use the TRAC method $[4,2]$ for redatuming the incident and scattered fields from $\Gamma$ to $\partial D_{S}$. Although, the time signature is unknown, the approximate locations of the source and the scatterer are required for both wave splitting and the TRAC method. 
Once all the needed information is available on a small neighborhood of $D_{S}$, enclosing the unknown scatterer $\mathcal{O}$, we perform the recovery by solving an inverse problem. Here, we opt for the Adaptive Eigenspace method [14, 13, 22, 25], which enables the inversion with only a few parameters.

In summary, we aim to recover the unknown inclusion $\mathcal{O}$ in three steps:

1. Wave splitting: extract the incident and the scattered wavefields from total field measurements

2. TRAC method: reconstruct separately the incident and the scattered wavefields in the vicinity of the obstacle

3. AEI method: solve the inverse problem from the redatumed total field

\subsection{Step 1: Retrieval of the incident and the scattered waves by wave splitting}

Our wave splitting method exploits absorbing boundary condition operators to isolate the incident and the scattered fields. Indeed both satisfy a transparent boundary condition, since both of them propagate to infinity [21]. The accuracy of low-order artificial boundary conditions is nonetheless sensitive to the incident angle of the propagating wavefield. We will use the reflectivity property of the artificial boundary condition to get the missing information about either the incident wavefield or the scattered wavefield.

Following [24], we consider the first-order absorbing boundary condition of Bayliss and Turkel [6]

$$
B[u]:=\frac{\partial u}{\partial t}+c \frac{\partial u}{\partial r}+c \frac{u}{2 r},
$$

which annihilates the leading order term in the asymptotic expansion in inverse powers of distance $r$ of an outgoing two-dimensional wavefield. Note that the accuracy of the boundary condition

$$
B[u]=0
$$

is intrinsically sensitive to the origin of the emitted signal due to the presence of the variable $r$. In particular, a wavefield impinging with a ninety-degree angle on the boundary will be almost perfectly absorbed, whereas the spurious reflection will be much larger at grazing incidence. By linearity, we have

$$
\begin{aligned}
& B_{I}[u]=B_{I}\left[u^{I}+u^{S}\right]=B_{I}\left[u^{I}\right]+B_{I}\left[u^{S}\right], \\
& B_{S}[u]=B_{S}\left[u^{I}+u^{S}\right]=B_{S}\left[u^{I}\right]+B_{S}\left[u^{S}\right],
\end{aligned}
$$

where the index $I$ indicates that the distance $r=r_{I}$ is taken from the origin $x_{\text {src }}$ of the source whereas the index $S$ corresponds to the approximate location of the obstacle $\mathcal{O}$.

Since each boundary condition is centered about the origin of its respective wavefield,

$$
B_{I}\left[u^{I}\right] \simeq 0 \quad \text { and } \quad B_{S}\left[u^{S}\right] \simeq 0 .
$$

Neglecting higher order terms, each considered wavefield satisfies

$$
\begin{aligned}
& B_{I}\left[u^{S}\right]=B_{I}[u], \\
& B_{S}\left[u^{I}\right]=B_{S}[u] .
\end{aligned}
$$

We are now able to retrieve $u^{I}$ and $u^{S}$ by solving equations (3) and (4), which we rewrite as

$$
\begin{aligned}
\frac{\partial u^{S}}{\partial t}+c \frac{\partial u^{S}}{\partial r_{I}}+c \frac{u}{2 r_{I}} & =\frac{\partial u}{\partial t}+c \frac{\partial u}{\partial r_{I}}+c \frac{u}{2 r_{I}}, \\
\frac{\partial u^{I}}{\partial t}+c \frac{\partial u^{I}}{\partial r_{S}}+c \frac{u}{2 r_{S}} & =\frac{\partial u}{\partial t}+c \frac{\partial u}{\partial r_{S}}+c \frac{u}{2 r_{S}} .
\end{aligned}
$$


Here, the incident field is described in polar coordinates centered at the obstacle's center of gravity, and the scattered field is described in polar coordinates centered at the source $x_{s r c}$.

Next, we express each equation in its corresponding coordinate system, for instance from $\left(r_{S}, \theta_{S}\right)$ to $\left(r_{I}, \theta_{I}\right)$ in $(6)$

$$
\begin{aligned}
\frac{\partial}{\partial r_{S}} & =\frac{r_{S}+\ell \cos \left(\theta_{S}\right)}{r_{I}} \frac{\partial}{\partial r_{I}}+\frac{\ell \sin \left(\theta_{S}\right)}{r_{I}^{2}} \frac{\partial}{\partial \theta_{I}} \\
\frac{\partial}{\partial r_{I}}\left(\sqrt{r_{I}} u^{I}\right) & =-\frac{1}{c} \frac{\partial}{\partial t}\left(\sqrt{r_{I}} u^{I}\right),
\end{aligned}
$$

with $\ell$ the distance between the source and the center of gravity of the obstacle.

Then, we rewrite (6) in terms of $f^{I}\left(r_{I}-c t, \theta_{I}\right):=\sqrt{r_{I}} u^{I}\left(t, r_{I} \cos \left(\theta_{I}\right), r_{I} \sin \left(\theta_{I}\right)\right)$ as

$$
\left(\alpha_{I}\left(\theta_{I}\right) \frac{\partial}{\partial t}+\beta_{I}\left(\theta_{I}\right) \frac{\partial}{\partial \theta_{I}}+\gamma_{I}\left(\theta_{I}\right)\right) f^{I}=\left(\frac{1}{c} \frac{\partial}{\partial t}+\frac{\partial}{\partial r_{S}}+\frac{1}{2 r_{S}}\right) u
$$

where the coefficients $\alpha_{I}, \beta_{I}$ and $\gamma_{I}$ in (7) are given by

$$
\begin{aligned}
\alpha_{I}\left(\theta_{I}\right) & =\frac{\sqrt{r_{I}^{2}+\ell^{2}-2 r_{I} \ell \cos \left(\theta_{I}\right)}-r_{I}+\ell \cos \left(\theta_{I}\right)}{c \sqrt{r_{I}} \sqrt{r_{I}^{2}+\ell^{2}-2 r_{I} \ell \cos \left(\theta_{I}\right)}}, \\
\beta_{I}\left(\theta_{I}\right) & =\frac{\ell \sin \left(\theta_{I}\right)}{r_{I} \sqrt{r_{I}} \sqrt{r_{I}^{2}+\ell^{2}-2 r_{I} \ell \cos \left(\theta_{I}\right)}}, \\
\gamma_{I}\left(\theta_{I}\right) & =\frac{\ell \cos \left(\theta_{I}\right)}{2 r_{1} \sqrt{r_{I}} \sqrt{r_{I}^{2}+\ell^{2}-2 r_{I} \ell \cos \left(\theta_{I}\right)}} .
\end{aligned}
$$

We note that (7) is a one-dimensional hyperbolic partial differential equation on $\Gamma$ for the unknown incident field $u^{I}=f^{I} / \sqrt{r_{I}}$. The signs of coefficients $\alpha_{I}, \beta_{I}$ and $\gamma_{I}$ determine how to integrate (7). We indeed observe that both $\alpha_{I}$ and $\beta_{I}$ vanish for $\theta_{I}=0$ or $\pi$, whereas $\gamma_{I}$ changes of sign at $\theta_{I}=$ $\pm \pi / 2$. Thus, we may compute the solution of (7) independently on each quarter circle. Moreover, the characteristics require a Dirichlet boundary condition at $\theta_{I}=0, \pi$. Finally, we choose the implicit Crank-Nicholson scheme in time and upwinding finite differences in space.

A similar equation can be derived from (5) to retrieve the scattered field $u^{S}$, see $[23,24]$ for further details.

\subsection{Step 2: Reconstruction with the TRAC method}

From the previous step, we have now extracted $u^{I}$ and $u^{S}$ on the outer boundary $\Gamma$. Still, we cannot yet solve the inverse problem inside $\Omega$ from the split measurements, since the source term remains unknown. To avoid the singularity around the unknown source, we shall solve the inverse problem in a subdomain, typically $D_{S}$ in Fig. 1 or 2 , by redatuming the total field close to $D_{S}$, which clearly does not include $x_{s r c}$. To create redatumed measurements, we wish to back-propagate the measured total field in $\Omega$. To do so, we actually propose to decompose the reconstruction by recomputing separately the incident and the scattered wavefields from the split measurements.

First, we back-propagate the incident wave from $\Gamma$ into the computational domain using time reversal $[18,17]$. In doing so, the time reversed incident field needs to vanish once it reaches the location $x_{s r c}$ of the source. To avoid the singularity at $x_{s r c}$ and absorb the shrinking incident field $u_{R}^{I}$, we remove a ball $D_{I}$ around $x_{s r c}$, see Fig. 2. At the inner artificial boundary of $D_{I}$, we impose time 
reversed absorbing condition (TRAC) $[3,4]$ to compute the time reversed wave $u_{R}^{I}$ in $\Omega \backslash D_{I}$ :

$$
\left\{\begin{aligned}
\frac{\partial^{2} u_{R}^{I}}{\partial t^{2}}-\nabla\left(c^{2} \nabla u_{R}^{I}\right) & =0, & & \text { in }(0, T) \times \Omega \backslash D_{I}, \\
u_{R}^{I} & =0, & & \text { in } \Omega \backslash D_{I} \text { at } t=0, \\
\frac{\partial u_{R}^{I}}{\partial t} & =0, & & \text { in } \Omega \backslash D_{I} \text { at } t=0, \\
u_{R}^{I} & =u_{\text {data }}^{I}, & & \text { on }(0, T) \times \Gamma, \\
\operatorname{TRAC}_{I}\left[u_{R}^{I}\right] & =0, & & \text { on }(0, T) \times \partial D_{I} .
\end{aligned}\right.
$$

The $\mathrm{TRAC}_{I}$ operator corresponds to a time-reversed first-order Bayliss-Turkel boundary condition:

$$
\operatorname{TRAC}_{I}\left[u_{R}\right]:=\frac{\partial u_{R}}{\partial t}+c \frac{\partial u_{R}}{\partial n_{I}}-c \frac{u_{R}}{2 r_{I}},
$$

where $n_{I}$ represents the inward normal derivative of $\Omega \backslash D_{I}$ at the boundary $\partial D_{I}, r_{I}$ is the radius of $D_{I}$ and the index $R$ indicates time reversal. It ensure that $D_{I}$ acts as a numerical sink without spurious reflection. The reconstruction of $u^{I}$ in $\Omega \backslash D_{I}$ does not involve the inclusion $\mathcal{O}$ in the velocity profile. The wave speed is assumed homogeneous, as it would be in our context for the computation of the forward incident wave.
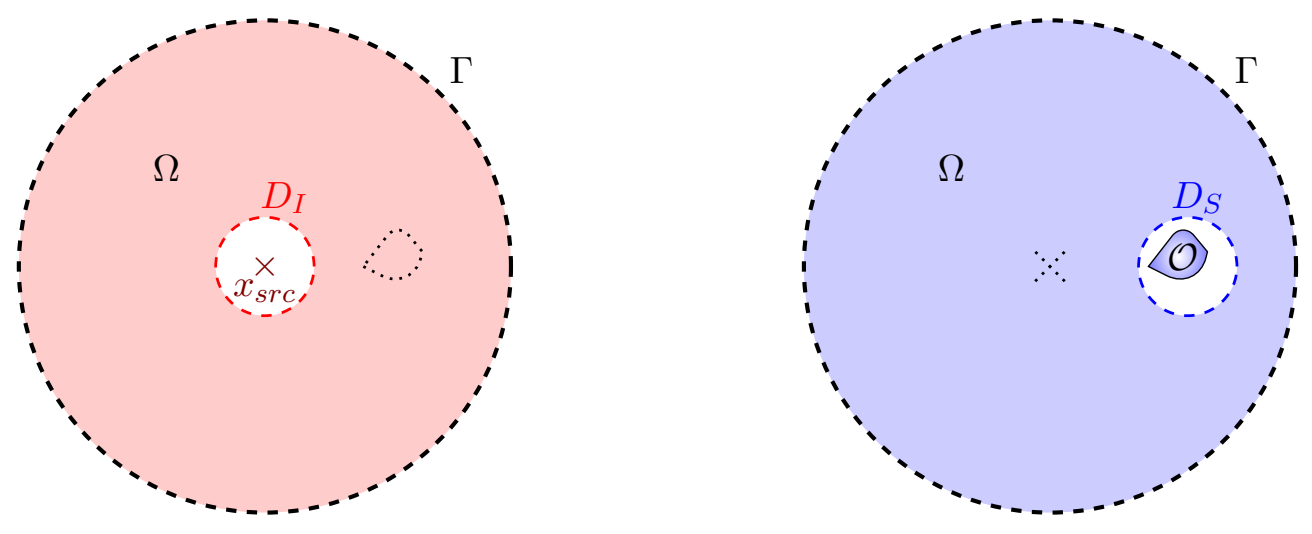

Figure 2: Reconstruction of the incident and scattered wavefields from the wave-split data on the boundary $\partial \Omega$ by performing the TRAC method for each wavefield: (left) for $u^{I}$, (right) for $u^{S}$. In both reconstructions, the computational domain is homogeneous, even though we marked the missing elements in dotted lines for reference.

Next, we reconstruct the scattered field using once more the TRAC method by removing a ball $D_{S}$ around the inclusion $\mathcal{O}$. Here again, no source is involved in the reconstruction of $u^{S}$. Similarly to (8), the time-reversed scattered field $u_{R}^{S}$ satisfies:

$$
\left\{\begin{aligned}
\frac{\partial^{2} u_{R}^{S}}{\partial t^{2}}-\nabla\left(c^{2} \nabla u_{R}^{S}\right) & =0, & & \text { in }(0, T) \times \Omega \backslash D_{S}, \\
u_{R}^{S} & =0, & & \text { in } \Omega \backslash D_{S} \text { at } t=0, \\
\frac{\partial u_{R}^{S}}{\partial t} & =0, & & \text { in } \Omega \backslash D_{S} \text { at } t=0, \\
u_{R}^{S} & =u_{d a t a}^{S}, & & \text { on }(0, T) \times \Gamma, \\
\operatorname{TRAC}_{S}\left[u_{R}^{S}\right] & =0, & & \text { on }(0, T) \times \partial D_{S},
\end{aligned}\right.
$$

where $n_{S}$ represents the inward normal derivative at $\partial D_{S}$ and $r_{S}$ is the radius of $D_{S}$ for the TRAC ${ }_{S}$ operator defined like in (9). 
Note that to redatum $u$ on $\partial D_{S}$, see Fig. 2, we could back-propagate $u$ directly to $\Omega \backslash\left(D_{S} \cup D_{I}\right)$ by imposing two numerical sinks in $D_{I}$ and $D_{S}$ [2]. However, this "naive $T R A C$ " approach proved not to be sufficiently accurate - see Section 3, because it ignores multiple transmissions and reflections between the two subdomains. Instead, we will perform the TRAC method on the incident wavefield $u^{I}$ in $\Omega \backslash D_{I}$ (resp. scattered wavefield $u^{S}$ in $\Omega \backslash D_{S}$ ) and sum them to get the total field $u^{I}+u^{S}$ in $\Omega \backslash\left(D_{I} \cup D_{S}\right)$, therefore on a small annulus around $D_{S}$ not containing $x_{s r c}$.

\subsection{Step 3: Recovery with Adaptive Eigenspace method}

The solution of the inverse problem consists in minimizing the functional $J$ :

$$
J(p)=\frac{1}{2}\left\|v(p)-u^{o b s}\right\|_{L^{2}((0, T) \times \omega)}^{2}
$$

where $v$ solves the wave equation

$$
\begin{aligned}
\frac{\partial^{2} v}{\partial t^{2}}-\nabla(p \nabla v) & =0, & & \text { in }(0, T) \times\left(\omega \cup D_{S}\right), \\
v & =0, & & \text { in }\left(\omega \cup D_{S}\right) \text { at } t=0, \\
\frac{\partial v}{\partial t} & =0, & & \text { in }\left(\omega \cup D_{S}\right) \text { at } t=0, \\
v & =u^{o b s,} & & \text { on }(0, T) \times \partial\left(\omega \cup D_{S}\right),
\end{aligned}
$$

and $u^{o b s}$ denotes the redatumed total field, acting as measurement data, in the observation set $\omega$, see Figure 3 , and $p=c^{2}$ the squared velocity known in $\omega$ but unknown inside $D_{S}$. Usually, the solution of the optimization problem is done using the standard local nodal basis from the chosen discretization scheme. One adds a regularization penalty term, typically Tikhonov or Total-Variation, to modify the optimization problem as:

$$
J(p)=\frac{1}{2}\left\|v(p)-u^{o b s}\right\|_{L^{2}((0, T) \times \omega)}^{2}+\frac{\alpha}{2} \mathcal{R}(p) .
$$

However, the choice of the added parameter $\alpha$ is crucial to allow the solution to reach the correct global minimum of the functional. The solution can be indeed very sensitive to the choice of $\alpha$. Moreover, to numerically solve the optimization problem, ones has to compute the Hessian matrix of functional $J$, for the Newton algorithm, for instance, or a good approximation of it, like the Gauss-Newton algorithm, both of which depending on $\mathcal{R}$.

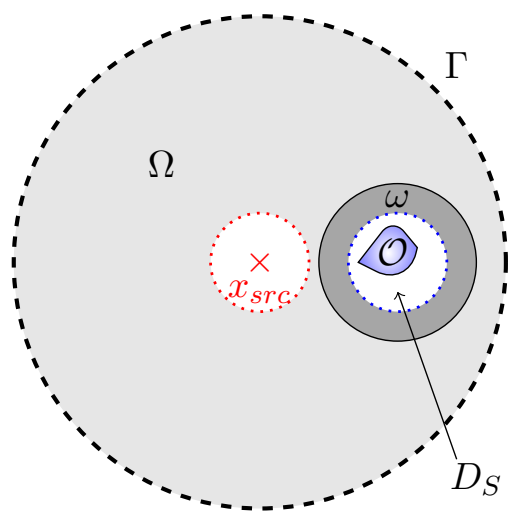

Figure 3: Computational domain $\left(\omega \cup D_{S}\right)$ for the inverse problem: from the reconstructed total field in $\Omega \backslash\left(D_{I} \cup D_{S}\right)$ we extract the redatumed data in $\omega$.

In the proposed alternative [13], we consider functional $J$ from (11) only. Now, the computation of the Hessian matrix, or approximation of it, does not depend on the smoothness of the chosen 
regularization functional $\mathcal{R}$. In this approach, we use the parametrization of the unknown parameter $p$, as suggested by the AEI method, i.e. with eigenfunctions of a well chosen elliptic operator. The idea of the Adaptive Eigenspace Inversion method is to expand the unknown control $p$ in a global basis:

$$
p(x)=p_{0}(x)+\sum_{m \geq 0} \beta_{m} \phi_{m}(x), \quad \forall x \in\left(\omega \cup D_{S}\right),
$$

where the basis functions $\phi_{m}$ are eigenfunctions of the elliptic problem:

$$
\left\{\begin{aligned}
\nabla \cdot\left(\mu(x) \nabla \phi_{m}\right) & =\lambda_{m} \phi_{m}, & & \text { in } D_{S} \\
\phi_{m} & =0, & & \text { in } \omega,
\end{aligned}\right.
$$

with

$$
\mu(x)=\frac{1}{|\nabla p(x)|+\varepsilon}, \quad \forall x \in D_{S}, \varepsilon>0 .
$$

and the boundary contribution satisfies the elliptic problem

$$
\left\{\begin{aligned}
\nabla \cdot\left(\mu(x) \nabla p_{0}\right) & =0, & & \text { in } D_{S} \\
p_{0} & =c^{2}, & & \text { in } \omega,
\end{aligned}\right.
$$

for the same weight $\mu$ from (15) and the known coefficient $p_{0}$ on the boundary; in fact, $p_{0}$ is constant in our examples.

The AEI method uses an iterative strategy: we first consider the eigenfunctions of the Laplacian operator as initial basis, since $p$ is constant, at the beginning. Then, we update $\mu$, the basis $\phi_{m}$ and the boundary contribution $p_{0}$ from the current $p$, before proceeding with the next iteration.

\section{AEI Algorithm.}

Input: initial guess $p=1$, observations $u^{o b s}$. Output: $p^{*}$.

1. Choose an initial value $K \geq 1$ and compute $\left\{\phi_{m}\right\}_{m=1}^{K}$ from (14) and $p_{0}$ from (16) with $\mu \equiv 1$

2. Expand $p(x)=p_{0}(x)+\sum_{m=1}^{K} \beta_{m} \phi_{m}(x)$

3. For $j=1, \ldots$

(a) Until tolerance is reached, solve the optimization problem with a truncated Quasi-Newton method to get an updated version of $p$

(b) Set $\mu$ from (15) with $\nabla p$

(c) Update $K$ and compute $\left\{\phi_{m}\right\}_{m=1}^{K}$ from (14) and $p_{0}$ from (16)

(d) Expand $p(x)=p_{0}(x)+\sum_{m=1}^{K} \beta_{m} \phi_{m}(x)$

4. $p^{*}=p$

In contrast to the algorithm presented in [22], here the AEI algorithm is actually designed to solve the time-dependent inverse problem. As a result, the number of iterations in the adaptive process is not dictated by the frequency range; here we choose to follow the idea of de Buhan and Kray in [13], which introduces only four iterations. However, unlike in [13] or [12], we do not change the formula describing the adaptive weight $\mu$ at each iteration, but instead vary the number of eigenfunctions $K$. In [13], this number remains indeed constant during the whole process and the optimization problem needed extra regularization as a result. Note that in the seminal work on AEI for the time-dependent viscoelastic equation by de Buhan and Osses [14], no extra regularization was added since the projection to the $K$ 
first eigenfunctions was already mentioned as the regularization method. Even though there is little theory yet about the choice of $K$ for the time-dependent case, we observe that the fewer eigenfunctions in the expansion, the smoother and coarser the reconstruction. Increasing $K$ is intuitively equivalent to decreasing $\alpha$ in Tikhonov type regularization and corresponds to relaxing the regularization after each iteration of the optimization. In addition to the varying number of basis functions, we use a truncated Quasi-Newton method [35, 36, 38], which acts as regularization here as well.

\section{$3 \quad$ Numerical examples}

In the following, we will illustrate each step of our approach through two numerical examples. We start with a simple shape, an ellipse, then test our method on a more angular shape, a fish. Both obstacles are penetrable with a $3: 1$ contrast in the squared velocity $c^{2}$. The duration of the experiment is $T=2$ and we set the recording array at a distance of $5 \lambda$ from the source, while the obstacle is at a distance of $2 \lambda$ from the source, where $\lambda$ denotes the wavelength. The main time-frequency of the source is $\nu=5$ and the size of the obstacle is about $\lambda / 2$.

For the forward simulation, we use a combination of Ricker signals. Computations of the synthetic data are performed with FreeFem $++[28]$ using $\mathbb{P}^{2}$ finite elements with a mesh size $h=\lambda / 20$ and the Crank-Nicholson scheme in time. On the external boundary at $15 \lambda$, we impose an absorbing boundary condition to ensure that no spurious reflections occur at the artificial boundary. As synthetic measurement data, we use the values of the forward total field and its normal derivative on the circle $\Gamma$ centered at $x_{s r c}$ with radius $5 \lambda$, see Fig. 4.

To show the accuracy of the method, we compare the time history of the retrieved wavefields with the true recorded ones at two different locations of the recording array $\Gamma$. We denote these two locations by their position with respect to the source: EastNorthEast and SouthWest, see Fig. 4.

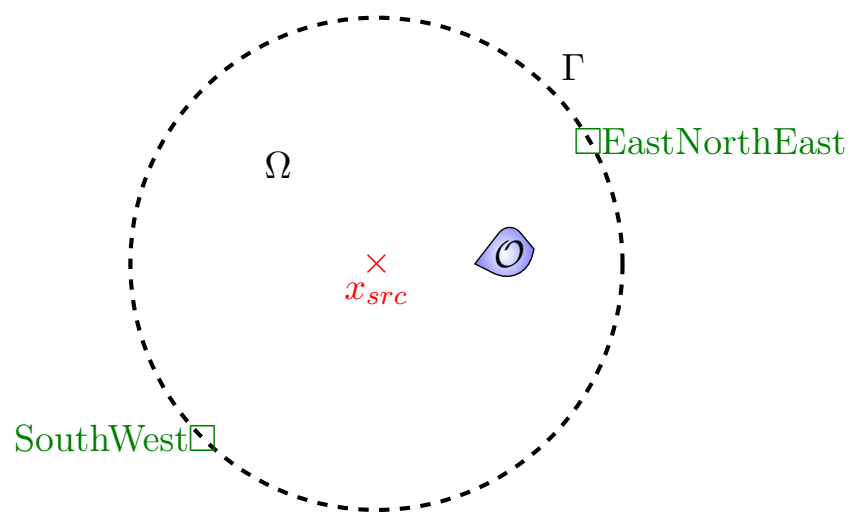

Figure 4: Retrieval of the incident and scattered components from the measurements on the boundary $\Gamma$ : two considered locations EastNorthEast and SouthWest. The source is located at $x_{s r c}$ and the obstacle $\mathcal{O}$ at 2 wavelengths from the source.

\subsection{Elliptic inclusion}

First, we consider an elliptic inclusion, with a 3:1 contrast in the squared wave propagation speed, which is quite low and difficult to detect. We have recorded the total field on the receiver's array located on the circular boundary $\Gamma$, centered at the approximate location of the source $x_{s r c}$ and with radius $5 \lambda$, see Fig. 4. 


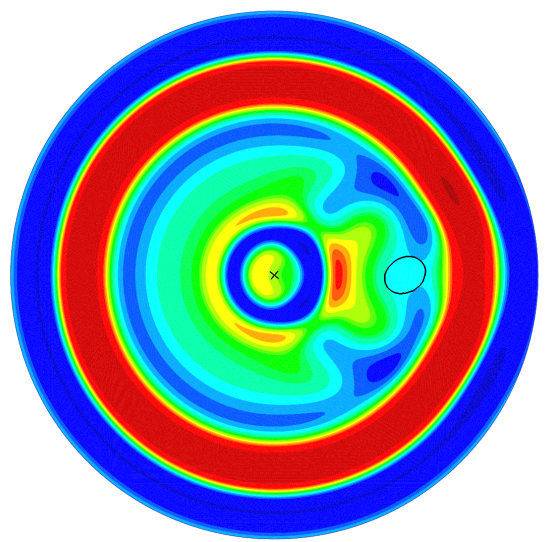

(a)

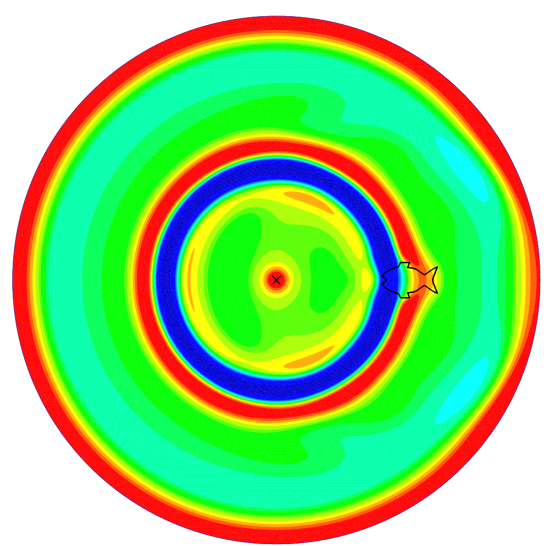

(b)

Figure 5: Snapshot of the synthetic total wavefield: source in the center, obstacle on the right of the source at $2 \lambda$, recording array at the boundary located at $5 \lambda$ from the source: (a) elliptic obstacle, (b) fish-shaped obstacle.

\subsubsection{Step 1: Wave Splitting}

To split the incident from the scattered field, we now perform our wave splitting algorithm described in Section 2.1 given the measured total wavefield and its normal derivative on $\Gamma$. We solve equations (3) and (4) on each quarter of a circle of $\Gamma$ by an upwinding finite differences scheme in $\theta$ and a CrankNicholson scheme in time, see [23, 24]. In Fig. 6 and 7, we display the time history retrieval at the two locations. In frame (a) we compare the retrieved incident wavefield $u^{I}$ with the reference field, as function of time $t$ at the specific locations. Similarly, frame (b) displays the retrieved scattered wavefield $u^{S}$ and the corresponding reference signal, while frame (c) compares their sum $u^{I}+u^{S}$ with the recorded total field $u$. Finally, in frame (d), we present the discrepancy between $u^{I}+u^{S}$ and $u$. Thus we observe that locally the reconstructions are really satisfactory. Moreover, the relative $L^{2}$-error (in time and space) in the computed wavefield is $0.57 \%$ only. Note that we avoid any potential inverse crime here since both the program and the numerical scheme differ from that used in the forward simulation.

\subsubsection{Step 2: TRAC method}

Having recovered the incident and scattered fields on boundary $\Gamma$, we now compute the time-reversed wavefields thanks to the TRAC method to reconstruct the wavefields inside the computational domain: $\Omega \backslash D_{I}$ for the incident wave $u^{I}$ and $\Omega \backslash D_{S}$ for the scattered field $u^{S}$. We recall that $u^{I}$ and $u^{S}$ are used for redatuming the total wavefield $u=u^{I}+u^{S}$ to later reduce the computational domain in the inverse problem algorithm.

Here, we use the software FreeFem $++[28]$ with $\mathbb{P}^{1}$ finite elements and the implicit Crank-Nicholson scheme in time. The current computational domain is an annulus of radius $5 \lambda$ with mesh size $h=\lambda / 10$, which again differs from the scheme used for the forward simulation thereby avoiding inverse crime. In Fig. 8, we show a snapshot of the total field obtained via four different computations:

(a) exact total field from the forward wave equation

(b) separate reconstruction of $u^{I}$ and $u^{S}$ with the TRAC method from the exact $u^{I}$ and $u^{S}$ recorded on the outer boundary $\Gamma$

(c) separate reconstruction of $u^{I}$ and $u^{S}$ with the TRAC method from $u^{I}$ and $u^{S}$ recovered on the outer boundary $\Gamma$ by wave splitting

(d) reconstruction of the total field directly through a naive TRAC method version with two sinks [2] 


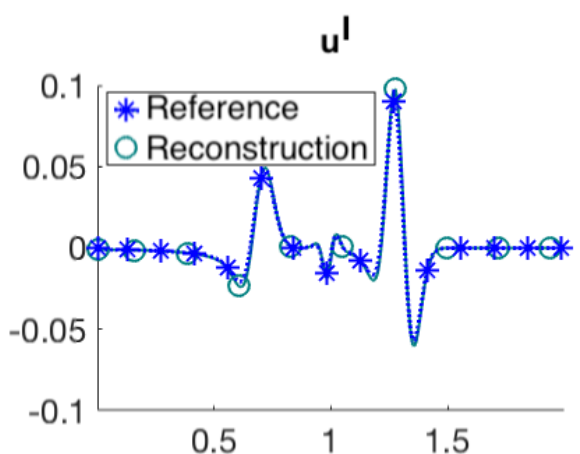

(a)

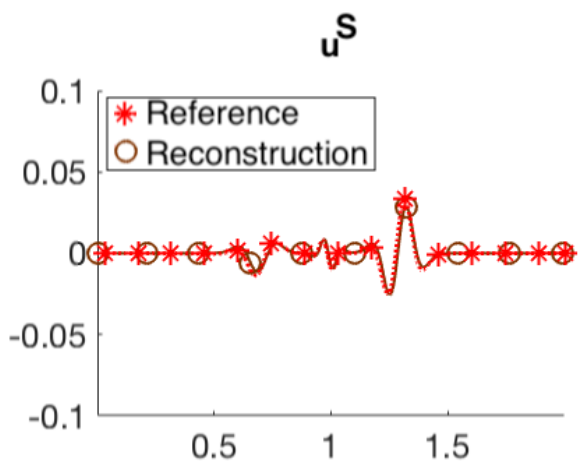

(c) u total

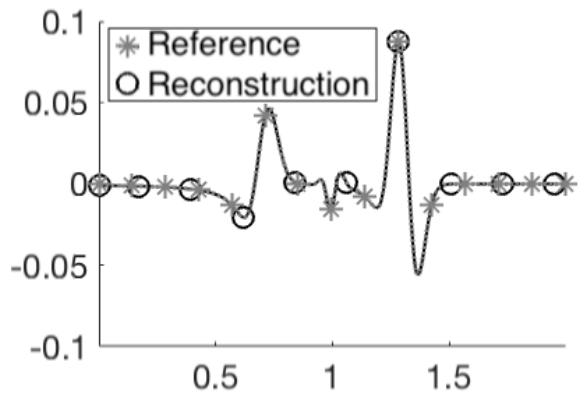

(b)

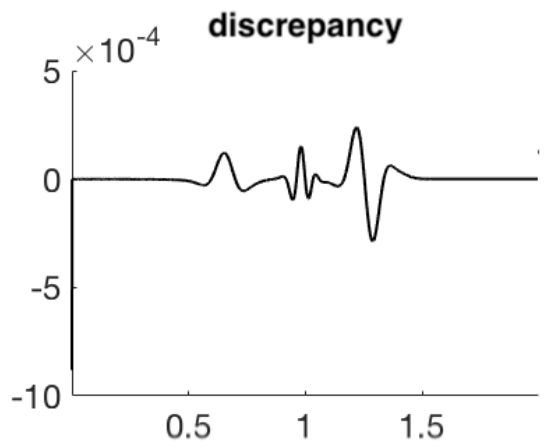

(d)

Figure 6: Time history retrieval at EastNorthEast for the elliptic obstacle: (a) incident wave, (b) scattered wave, (c) total field and (d) discrepancy between the measured total field and the sum of the retrieved incident and scattered fields.

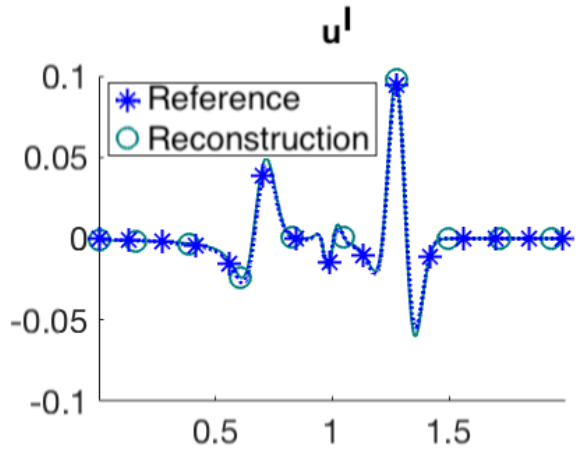

(a)

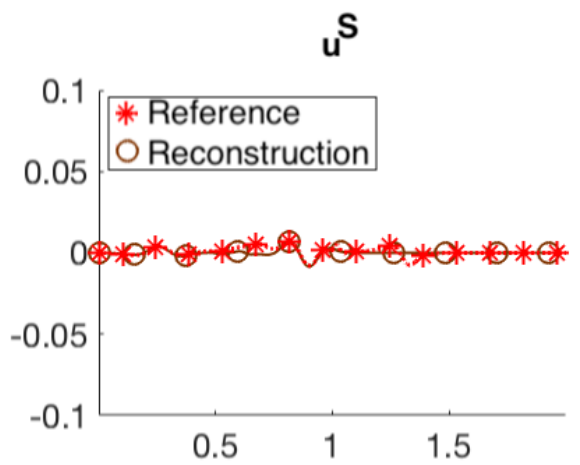

(c) u total

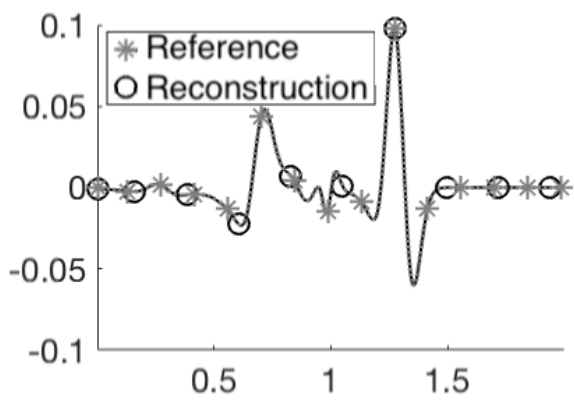

(b)

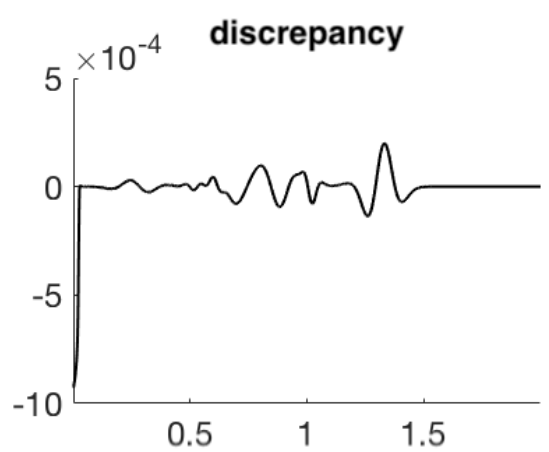

(d)

Figure 7: Time history retrieval at SouthWest for the elliptic obstacle: (a) incident wave, (b) scattered wave, (c) total field and (d) discrepancy between the measured total field and the sum of the retrieved incident and scattered fields. 
We observe that our reconstruction of the total wavefield from the recovered $u^{I}$ and $u^{S}$ is satisfactory: about $5 \%$ of $L^{2}$-error (in time and space) versus $25 \%$ for the naive $T R A C$ version, see Table 1 . Clearly, by reconstructing $u^{I}$ and $u^{S}$ separately with $T R A C$, we obtain a much better reconstruction of the total field than if we only consider the naive TRAC version, since it takes into account the transmissions between $D_{I}$ and $D_{S}$. However, we can also notice that the error in the reconstruction is bigger between the source and the obstacle (in $\omega$ ), where the information for the inverse problem is needed.

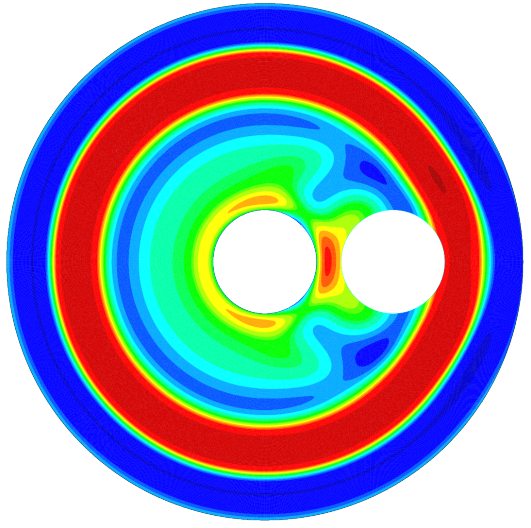

(a)

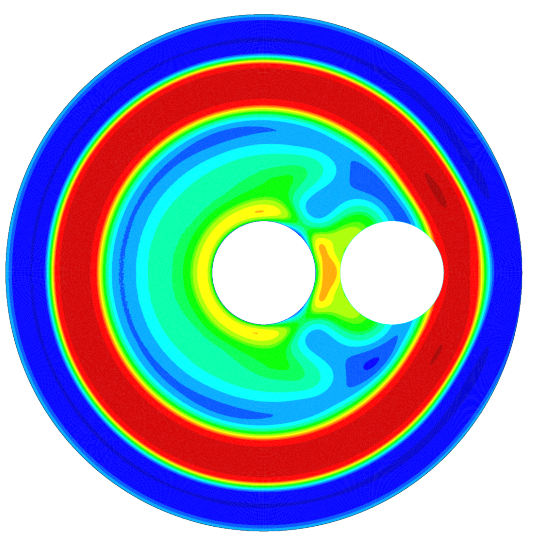

(c)

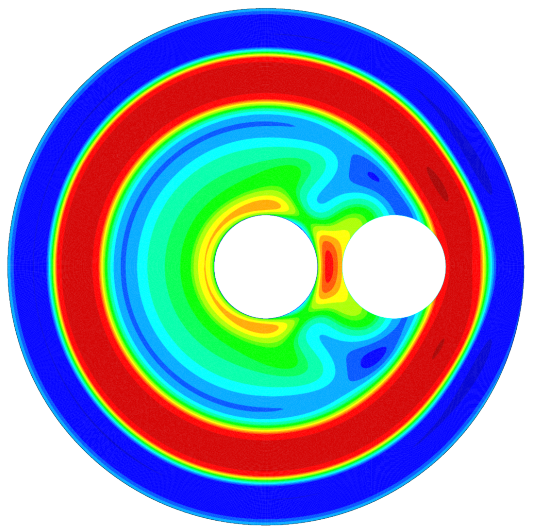

(b)

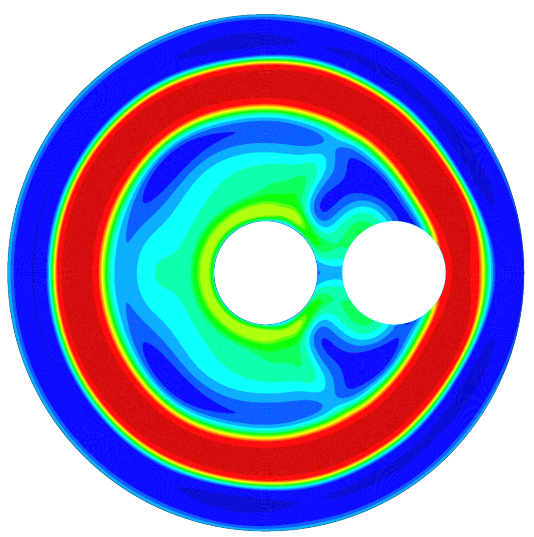

(d)

Figure 8: Elliptic obstacle: Snapshot of the reconstruction of the total field $u=u^{I}+u^{S}$ with the TRAC method. (a) Exact total field $u$ for reference. (b) From the exact (recorded for reference) $u^{I}$ and $u^{S}$. (c) From $u^{I}$ and $u^{S}$ obtained with wave splitting. (d) Naive TRAC with two sinks.

\begin{tabular}{c|cc} 
& $\begin{array}{c}\text { relative } L^{2} \text {-error } \\
\text { in }(0, T) \times\left(\Omega \backslash\left(D_{I} \cup D_{S}\right)\right)\end{array}$ & $\begin{array}{c}\text { relative } L^{2} \text {-error } \\
\text { in }(0, T) \times \omega\end{array}$ \\
\hline$u^{I}+u^{S}$ from exact measurements & $4.52 \%$ & $6.27 \%$ \\
$u^{I}+u^{S}$ from wave splitting & $5.71 \%$ & $8.02 \%$ \\
total $u$ from naive TRAC & $24.99 \%$ & $43.76 \%$
\end{tabular}

Table 1: Elliptic obstacle: Reconstruction of the total wavefield with the TRAC method. Relative $L^{2}$-error comparing the forward total wavefield (Fig. 8(a)) and the reconstructed wavefields: From the exact (recorded for reference) $u^{I}$ and $u^{S}$ (Fig. 8(b)), from $u^{I}$ and $u^{S}$ obtained by wave splitting (Fig. 8(c)), and $u$ using the naive TRAC approach with two sinks (Fig. 8(d)). 


\subsubsection{Step 3: AEI}

Finally, we solve the inverse problem in $D_{S}$ from the reconstructed and redatumed data in $\omega$ using the AEI method. As in [22], we solve the optimization problem using the truncated BFGS method with only a few CG iterations to solve the Newton step. In doing so, we adapt four times the eigenfunctions basis and its dimension. We start with 20 eigenfunctions of the Laplacian, then update the weight $\mu$ in (15) and use 50, 100 and finally 100 eigenfunctions for iteration 2, 3 and 4 respectively of the AEI algorithm. All computations are performed with FreeFem ++ with $\mathbb{P}^{1}$ finite elements for computational domain $\omega \cup D_{S}$ with a mesh size $h=\lambda / 10$ and the Crank-Nicholson time scheme. As the computational mesh is totally different from those used in the forward simulation and in the TRAC reconstruction, we ensure here that there is no inverse crime.

In Fig. 9, we compare the recovered parameter $p$ obtained either by solving the inverse problem with (a) the exact data and (b) the data from our three-step strategy. The location, the shape and the contrast are almost as accurate as if the exact data is used. Note that both pictures are displayed with the same scale for better comparison.
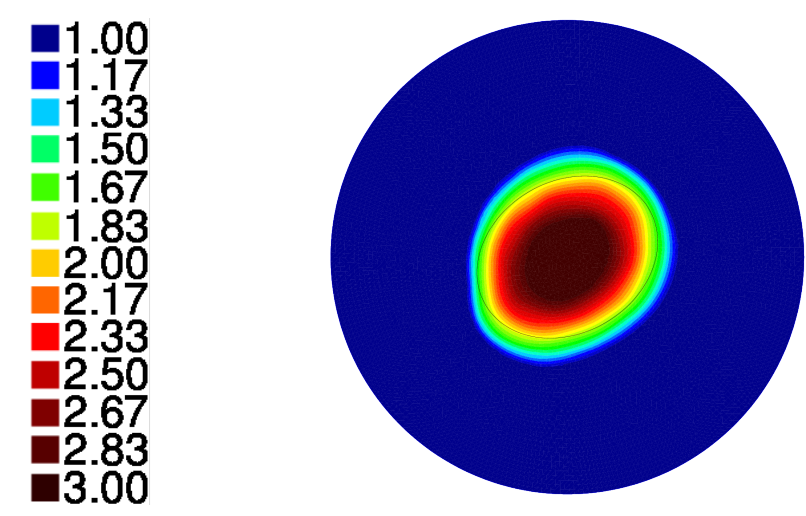

(a)

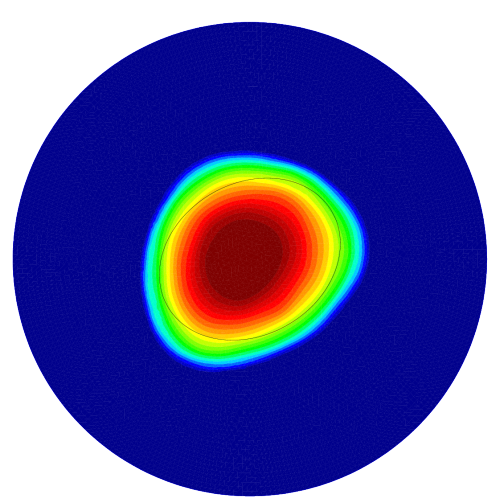

(b)

Figure 9: Recovery of $p=c^{2}$ for the elliptic obstacle $\mathcal{O}$ with the AEI method: (a) from the exact total field, (b) in our three-step strategy.

\section{$3.2 \quad$ Fish-shaped obstacle}

Next, we consider a fish-shaped inclusion with 3:1 contrast in the squared wave speed. The total field is recorded on the receiver's array located on boundary $\Gamma$, as in Fig. 4.

\subsubsection{Wave Splitting}

Again, we perform our wave splitting algorithm by solving equations (3) and (4) by a finite differences. In Fig. 10 and 11, we display the time history retrieval at the two considered locations. Comparison of the computed wavefield with the reference yields a relative $L^{2}$-error of only $0.93 \%$ for the sum of the two individually split wavefields.

\subsubsection{TRAC method}

Having recorded the incident and scattered fields on the boundary, we now compute the time-reversed wavefields by the TRAC method to reconstruct the wavefields inside the computational domain. In Fig. 12, we show a snapshot of the total field obtained via four different computations:

(a) exact total field from the forward wave equation 


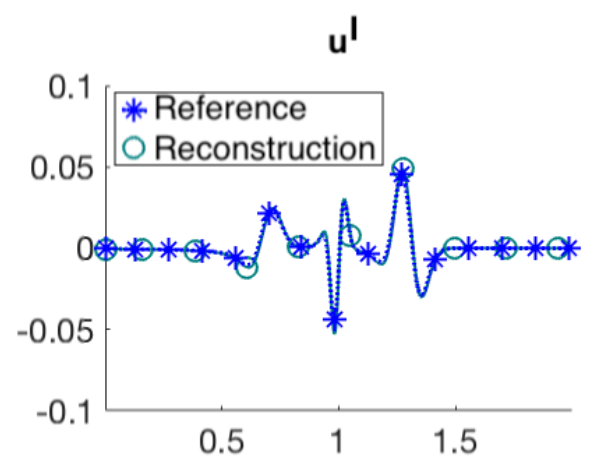

(a)

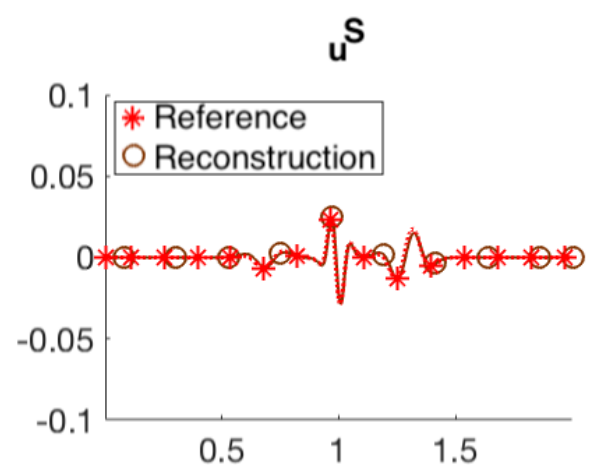

(c)

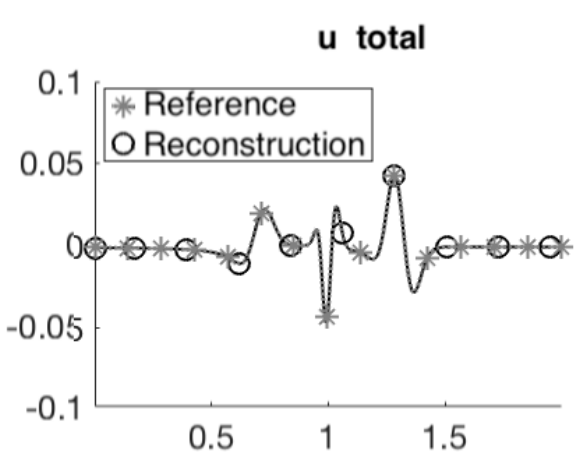

(b)

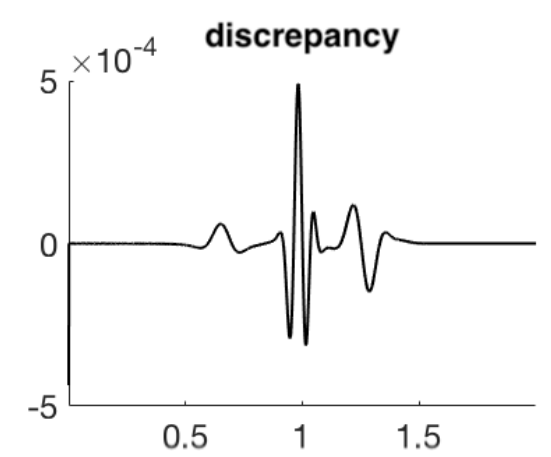

(d)

Figure 10: Time history retrieval at EastNorthEast for the fish-shaped obstacle: (a) incident wave, (b) scattered wave, (c) total field and (d) discrepancy between the measured total field and the sum of the retrieved incident and scattered fields.

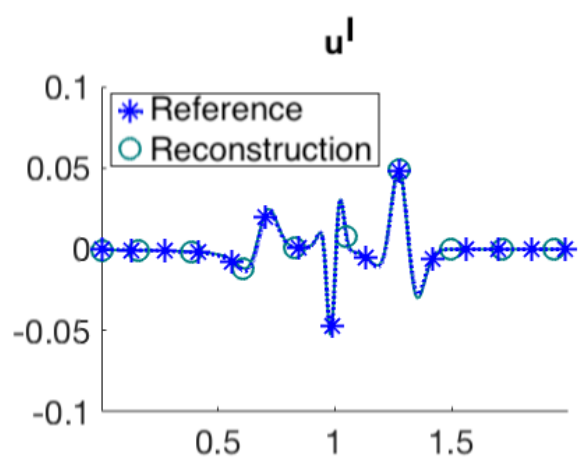

(a)

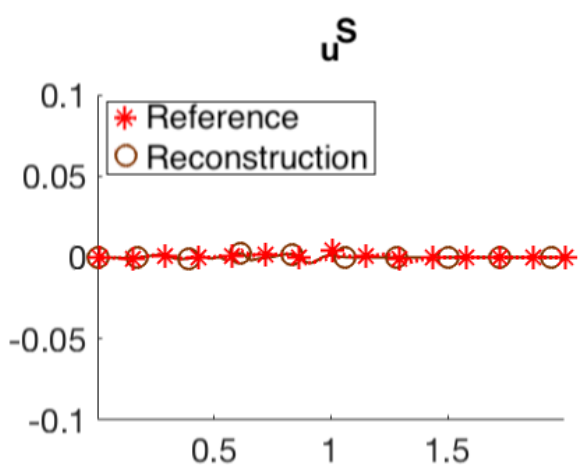

(c) u total

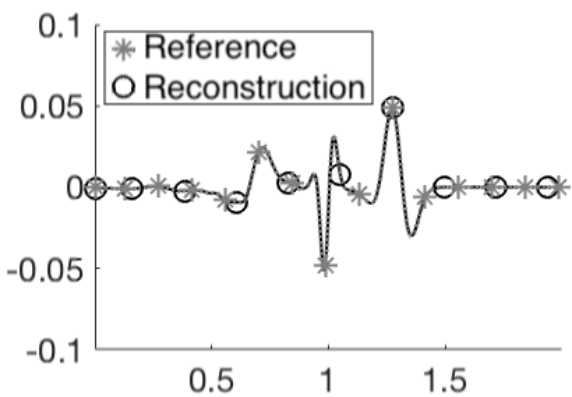

(b)

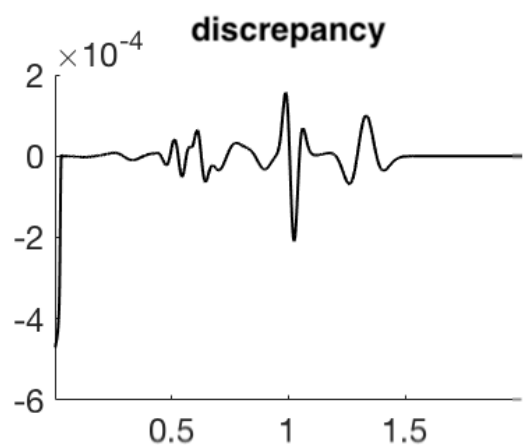

(d)

Figure 11: Time history retrieval at SouthWest for the fish-shaped obstacle: (a) incident wave, (b) scattered wave, (c) total field and (d) discrepancy between the measured total field and the sum of the retrieved incident and scattered fields 
(b) separate reconstruction of $u^{I}$ and $u^{S}$ with the TRAC method from the exact $u^{I}$ and $u^{S}$ recorded on the outer boundary

(c) separate reconstruction of $u^{I}$ and $u^{S}$ with the TRAC method from $u^{I}$ and $u^{S}$ recovered on the outer boundary by wave splitting

(d) reconstruction of the total field directly through a naive TRAC method version with two sinks [?]

The reconstruction of the total wavefield from the recovered $u^{I}$ and $u^{S}$ is quite satisfactory: about $6 \%$ of $L^{2}$-error (in time and space) versus $25 \%$ for the naive TRAC version, see Table 2 , actually comparable to the easier case of the ellipse. Clearly, by reconstructing $u^{I}$ and $u^{S}$ separately with $T R A C$, we again obtain a much better reconstruction of the total field than if we only consider the naive $T R A C$ version with two sinks.

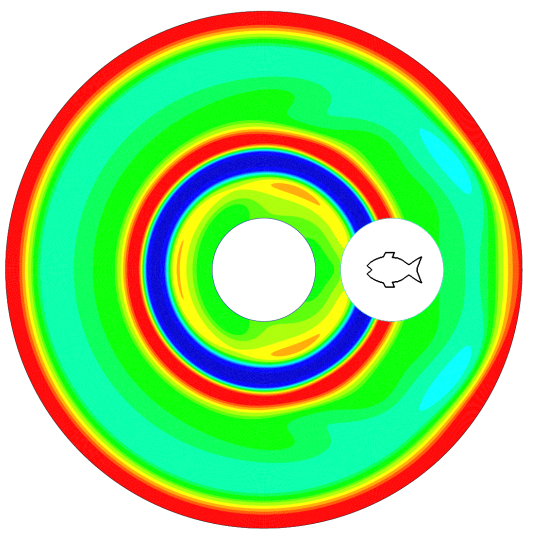

(a)

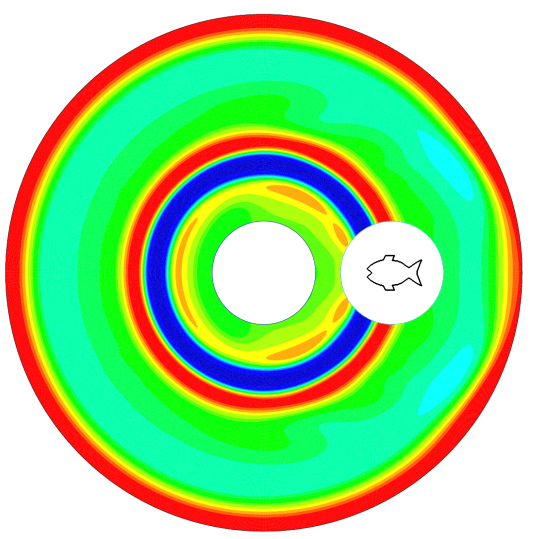

(c)

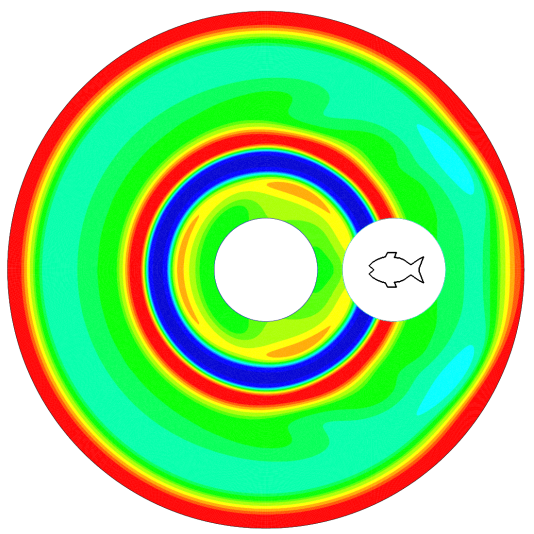

(b)

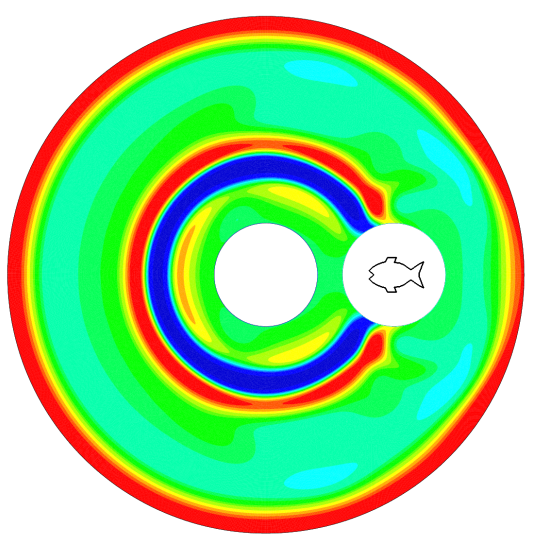

(d)

Figure 12: Fish-shaped obstacle: Snapshot of the reconstruction of the total field $u=u^{I}+u^{S}$ with the TRAC method. (a) Exact total field $u$ for reference. (b) From the exact (recorded for reference) $u^{I}$ and $u^{S}$. (c) From $u^{I}$ and $u^{S}$ obtained with wave splitting. (d) Naive TRAC with two sinks.

\subsubsection{AEI}

Finally, we solve the inverse problem in $D$ from the reconstructed data in $\omega$ using the AEI method. Similarly to the previous example, we solve the optimization problem using a truncated Quasi-Newton method while increasing the number of eigenfunctions at each new adaptation. We start with 20 eigenfunctions of the Laplacian, then update the weight $\mu$ in (15) using 50, 100 and 100 eigenfunctions 


\begin{tabular}{c|cc} 
& $\begin{array}{c}\text { relative } L^{2} \text {-error } \\
\text { in }(0, T) \times\left(\Omega \backslash\left(D_{I} \cup D_{S}\right)\right)\end{array}$ & $\begin{array}{c}\text { relative } L^{2} \text {-error } \\
\text { in }(0, T) \times \omega\end{array}$ \\
\hline$u^{I}+u^{S}$ from exact measurements & $3.99 \%$ & $5.66 \%$ \\
$u^{I}+u^{S}$ from wave splitting & $6.05 \%$ & $8.44 \%$ \\
total $u$ from naive TRAC & $24.07 \%$ & $42.10 \%$
\end{tabular}

Table 2: Fish-shaped obstacle: Reconstruction of the total wavefield with the TRAC method. Relative $L^{2}$-error comparing the forward total wavefield (Fig. 12(a)) and the reconstructed wavefields: From the exact (recorded for reference) $u^{I}$ and $u^{S}$ (Fig. 12(b)), from $u^{I}$ and $u^{S}$ obtained by wave splitting (Fig. 12(c)), and $u$ using the naive TRAC approach with two sinks (Fig. 12(d)).

for iteration 2, 3 and 4 respectively of the AEI algorithm. In Fig. 13, we compare the recovered parameter $p=c^{2}$ with (a) the exact observation data and (b) the data from our three-step strategy. The location is well recovered, despite a tail in the shadow zone of the fish, though the contrast and the shape are much harder to recover, since the obstacle is only about one wavelength long, so at the limit of resolution of inverse problem.
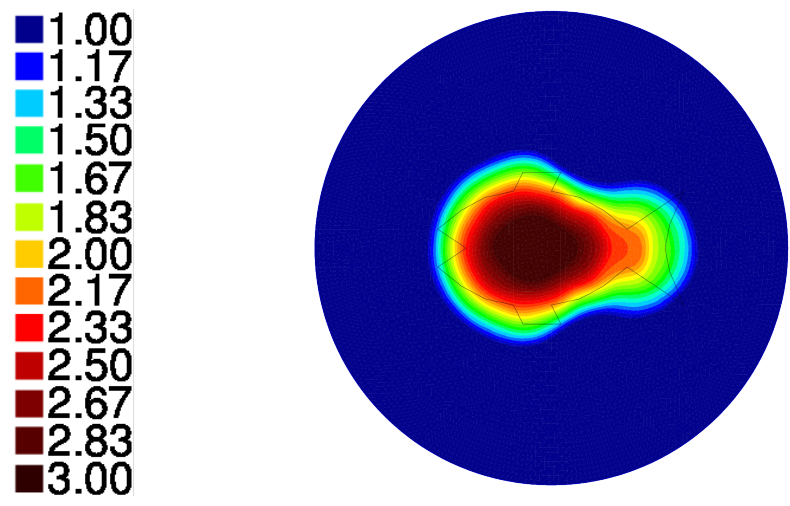

(a)

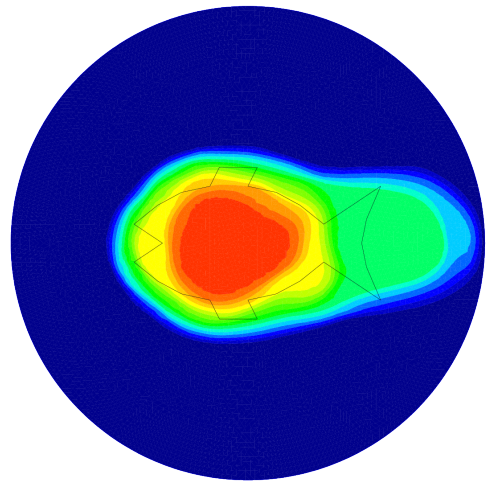

(b)

Figure 13: Recovery of $p=c^{2}$ for the fish-shaped obstacle $\mathcal{O}$ with the AEI method: (a) from the exact total field, (b) in our three-step strategy.

\section{Conclusion}

We aimed at solving an inverse scattering problem when the source signature was unknown. We therefore presented a three-step strategy that allowed us to overcome the problem of the knowledge of the source term by redatuming the measurement data into a zone close to the inclusion and avoiding the source location. To do so, we first retrieved the incident and the scattered wavefields separately from the measured total field by wave splitting. Then we reconstructed the incident and the scattered fields in the computational domain with the TRAC method. We showed that redatuming was more successful by reconstructing the incident and scattered fields individually and calculating their sum. We were then able to reduce the computational domain for the inverse problem by considering the redatumed total wavefield close to the inclusion. Finally, we solved the resulting inverse problem with Adaptive Eigenspace Inversion for two numerical examples. Although our methods and numerical examples are in two-space dimensions, they can be extended to 3D or other types of wave equations from electromagnetism or viscoelasticity. 


\section{Acknowledgments}

M. Graff was partially supported by the Swiss National Science Foundation grant P300P2-167681.

\section{References}

[1] S. Acosta. On-surface radiation condition for multiple scattering of waves. Comput. Meth. Appl. Mech. Engrg., 283:1296-1309, 2015.

[2] F. Assous, M. Kray, and F. Nataf. Time Reversed Absorbing Condition in the Partial Aperture Case. Wave Motion, 49(7):617-63, 2012.

[3] F. Assous, M. Kray, F. Nataf, and E. Turkel. Time reversed absorbing conditions. Comptes Rendus Mathematiques, 348(19-20):1063-1067, 2010.

[4] F. Assous, M. Kray, F. Nataf, and E. Turkel. Time Reversed Absorbing Condition: Application to inverse problem. Inverse Problems, 27(6):065003, 2011.

[5] D. H. Baffet and M. J. Grote. On wave splitting, source separation and echo removal with absorbing boundary conditions. J. Comp. Physics. In press.

[6] A. Bayliss and E. Turkel. Radiation boundary conditions for wave-like equations. Comm. Pure Appl. Math., 33(6):707-725, 1980.

[7] F. Ben Hassen, J. Liu, and R. Potthast. On source analysis by wave splitting with applications in inverse scattering of multiple obstacles. J. Comput. Math, 25(3):266-281, 2007.

[8] F. Cakoni and D. L. Colton. Qualitative methods in inverse scattering theory: an introduction. Interaction of mechanics and mathematics series. Springer, 2006.

[9] A. Chambolle, V. Duval, G. Peyré, and C. Poon. Geometric properties of solutions to the total variation denoising problem. Inverse Problems, 33(1):015002, 2017.

[10] H. Chang, Y. Lou, Y. Duan, and S. Marchesini. Total variation-based phase retrieval for Poisson noise removal. SIAM J. Imaging Sciences, 11(1):24-55, 2018.

[11] D. Colton and R. Kress. Inverse acoustic and electromagnetic scattering theory, volume 93 of Applied Mathematical Sciences. Springer-Verlag, New-York, second edition, 1998.

[12] M. de Buhan and M. Darbas. Numerical resolution of an electromagnetic inverse medium problem at fixed frequency. Computers and Mathematics with Applications, 74(12):3111-3128, 2017.

[13] M. de Buhan and M. Kray. A new approach to solve the inverse scattering problem for waves: combining the TRAC and the Adaptive Inversion methods. Inverse Problems, 29(8):085009, 2013.

[14] M. de Buhan and A. Osses. Logarithmic stability in determination of a 3D viscoelastic coefficient and a numerical example. Inverse Problems, 26(9):95006, 2010.

[15] I. Epanomeritakis, V. Akçelik, O. Ghattas, and J. Bielak. A Newton-CG method for large-scale three-dimensional elastic full-waveform seismic inversion. Inverse Problems, 24(3):034015, 2008.

[16] E. Esser, L. Guasch, T. van Leeuwen, and F. J. Herrmann. Total-variation regularization strategies in full-waveform inversion. SIAM J. Imaging Sci., 11(1):376-406, 2018.

[17] M. Fink. Time-reversal mirrors. J. Phys. D: Appl. Phys., 26(9):1333, 1993. 
[18] M. Fink. Renversement du temps, ondes et innovation. 2009. Collection Collège de France.

[19] R. Griesmaier, M. Hanke, and J. Sylvester. Far field splitting for the Helmholtz equation. SIAM J. Numer. Anal., 52(1):343-362, 2014.

[20] M. J. Grote and C. Kirsch. Dirichlet-to-Neumann boundary conditions for multiple scattering problems. J. Comput. Phys., 201(2):630-650, 2004.

[21] M. J. Grote and C. Kirsch. Nonreflecting boundary condition for time-dependent multiple scattering. J. Comput. Phys., 221(1):41-67, 2007.

[22] M. J. Grote, M. Kray, and U. Nahum. Adaptive eigenspace method for inverse scattering problems in the frequency domain. Inverse Problems, 33(2):025006, 2017.

[23] M. J. Grote, M. Kray, F. Nataf, and F. Assous. Wave splitting for time-dependent scattered field separation. Comptes Rendus Mathematiques, 353(6):523-527, 2015.

[24] M. J. Grote, M. Kray, F. Nataf, and F. Assous. Time-dependent wave splitting and source separation. J. Comput. Phys., 330:981-996, 2017.

[25] M. J. Grote and U. Nahum. Adaptive eigenspace for multi-parameter inverse scattering problems. Comp. Math. Applic. In press.

[26] E. Haber, U. M. Ascher, and D. Oldenburg. On optimization techniques for solving nonlinear inverse problems. Inverse Problems, 16:1263, 2000.

[27] H. Haddar, A. Lechleiter, and S. Marmorat. An improved time domain linear sampling method for Robin and Neumann obstacles. Applicable Analysis, 0:1-22, 2013.

[28] F. Hecht. New development in FreeFem++. J. Numer. Math., 20(3-4):251-265, 2012.

[29] M. Hinze, R. Pinnau, M. Ulbrich, and S. Ulbrich. Optimization with PDE Constraints, volume 23 of Mathematical Modelling: Theory and Applications. Springer Netherlands, first edition, 2009.

[30] A. Kirsch. The MUSIC-algorithm and the factorization method in inverse scattering theory for inhomogeneous media. Inverse Problems, 18(4):1025-1040, 2002.

[31] A. Kirsch. An Introduction to the Mathematical Theory of Inverse Problems, volume 120 of Applied Mathematical Sciences. Springer-Verlag New York, 2 edition, 2011.

[32] A. Lechleiter. The factorization method is independent of transmission eigenvalues. Inverse Problems and Imaging, 3(1):123-138, 2009.

[33] A. Lechleiter, K. S. Kazimierski, and M. Karamehmedović. Tikhonov regularization in $L^{p}$ applied to inverse medium scattering. Inverse Problems, 29(7):075003, 2013.

[34] A. Lechleiter and M. Rennoch. Non-linear Tikhonov regularization in Banach spaces for inverse scattering from anisotropic penetrable media. Inverse Problems and Imaging, 11(1):151-176, 2017.

[35] A. Lechleiter and A. Rieder. Newton regularizations for impedance tomography: a numerical study. Inverse Problems, 22(6):1967, 2006.

[36] A. Lechleiter and A. Rieder. Newton regularizations for impedance tomography: convergence by local injectivity. Inverse Problems, 24(6):065009, 2008.

[37] F. Lucka, N. Huynh, M. Betcke, E. Zhang, P. Beard, B. Cox, and S. Arridge. Enhancing compressed sensing $4 \mathrm{D}$ photoacoustic tomography by simultaneous motion estimation. SIAM J. Imaging Sciences, 11(4):2224-2253, 2018. 
[38] L. Métivier, R. Brossier, J. Virieux, and S. Operto. Full Waveform Inversion and the Truncated Newton Method. SIAM J. Sci. Comput., 35(2):B401-B437, 2013.

[39] P. Mojabi and J. LoVetri. Microwave Biomedical Imaging Using the Multiplicative Regularized Gauss-Newton Inversion. Antennas and Wireless Propagation Letters, IEEE, 8:645-648, 2009.

[40] U. Nahum and P. C. Cattin. Adaptive eigenspace segmentation. arXiv:1810.12771v1, 2018.

[41] M. Pernot, G. Montaldo, M. Tanter, and M. Fink. 'Ultrasonic stars' for time reversal focusing using induced cavitation bubbles. Appl. Phys. Lett., 88(3):034102, 2006.

[42] R. Potthast. A survey on sampling and probe methods for inverse problems. Inverse Problems, 22(2):R1, 2006.

[43] R. Potthast, F. M. Fazi, and P. A. Nelson. Source splitting via the point source method. Inverse Problems, 26(4):045002, 2010.

[44] T. Saratoon, T. Tarvainen, B. T. Cox, and S. R. Arridge. A gradient-based method for quantitative photoacoustic tomography using the radiative transfer equation. Inverse Problems, 29(7):075006, 19, 2013.

[45] A. Tarantola. Inverse Problem Theory And Methods For Model Parameter Estimation. Society for Industrial and Applied Mathematics, 2005.

[46] A. N. Tikhonov. On the stability of inverse problems. Dokl. Akad. Nauk SSSR, 39(5):195-198, 1943.

[47] V. Twersky. On multiple scattering of waves. J. Res. Nat. Bur. Standards, 64D:715-730, 1960.

[48] J. F. M. Van Doren, P. M. J. Van den Hof, J. D. Jansen, and O. H. Bosgra. Parameter identification in large-scale models for oil and gas production. In Proc. 18th IFAC World Congress, pages 1085710862, Milano, Italy, 2011. 